\title{
Liver $L X R \alpha$ expression is crucial for whole body cholesterol homeostasis and reverse cholesterol transport in mice
}

\author{
Yuan Zhang,, ${ }^{1}$ Sarah R. Breevoort, ${ }^{2}$ Jerry Angdisen,,2 Mingui Fu, ${ }^{1}$ Daniel R. Schmidt, ${ }^{1}$ \\ Sam R. Holmstrom, ${ }^{1}$ Steven A. Kliewer, ${ }^{1,3}$ David J. Mangelsdorf,1,4 and Ira G. Schulman² \\ ${ }^{1}$ Department of Pharmacology, University of Texas Southwestern Medical Center, Dallas, Texas, USA. ${ }^{2}$ Department of Pharmacology, \\ University of Virginia Health System, Charlottesville, Virginia, USA. ${ }^{3}$ Department of Molecular Biology and ${ }^{4}$ Howard Hughes Medical Institute, \\ University of Texas Southwestern Medical Center, Dallas, Texas, USA.
}

\begin{abstract}
Liver $X$ receptors ( $L X R \alpha$ and $L X R \beta)$ are important regulators of cholesterol and lipid metabolism, and their activation has been shown to inhibit cardiovascular disease and reduce atherosclerosis in animal models. Small molecule agonists of LXR activity are therefore of great therapeutic interest. However, the finding that such agonists also promote hepatic lipogenesis has led to the idea that hepatic LXR activity is undesirable from a therapeutic perspective. To investigate whether this might be true, we performed gene targeting to selectively delete LXR $\alpha$ in hepatocytes. Liver-specific deletion of LXR $\alpha$ in mice substantially decreased reverse cholesterol transport, cholesterol catabolism, and cholesterol excretion, revealing the essential importance of hepatic LXR $\alpha$ for whole body cholesterol homeostasis. Additionally, in a pro-atherogenic background, liverspecific deletion of LXR $\alpha$ increased atherosclerosis, uncovering an important function for hepatic LXR activity in limiting cardiovascular disease. Nevertheless, synthetic LXR agonists still elicited anti-atherogenic activity in the absence of hepatic LXR $\alpha$, indicating that the ability of agonists to reduce cardiovascular disease did not require an increase in cholesterol excretion. Furthermore, when non-atherogenic mice were treated with synthetic LXR agonists, liver-specific deletion of LXR $\alpha$ eliminated the detrimental effect of increased plasma triglycerides, while the beneficial effect of increased plasma HDL was unaltered. In sum, these observations suggest that therapeutic strategies that bypass the liver or limit the activation of hepatic LXRs should still be beneficial for the treatment of cardiovascular disease.
\end{abstract}

\section{Introduction}

The precise regulation of cholesterol metabolism is essential, and it is well known that elevated levels of cholesterol in the blood are a major cause of cardiovascular disease (1). Studies using global genetic knockouts and synthetic agonists have defined important roles for the liver X receptors LXR $\alpha(\mathrm{NR} 1 \mathrm{H} 3)$ and LXR $\beta(\mathrm{NR} 1 \mathrm{H} 2)$ in the control of cholesterol metabolism (2). LXRs are members of the nuclear hormone receptor superfamily of ligand-activated transcription factors, and treatment of animals with LXR agonists results in changes in gene expression promoting the efflux of cholesterol from peripheral cells such as macrophages, the excretion of cholesterol from the liver, and the inhibition of cholesterol absorption in the intestine (2). Importantly, the endogenous ligands for LXRs are oxidized forms of cholesterol (oxysterols) (3, 4) that increase coordinately with intracellular cholesterol levels, thus allowing these receptors to function as sensors to maintain cholesterol at appropriate levels throughout the body.

At the molecular level, LXRs control cholesterol efflux by regulating expression of the genes encoding the ATP-binding cassette (ABC) transporters ABCA1 and ABCG1 (2). Upregulation of ABCA1 and ABCG1 results in increased transfer of intracellular cholesterol to HDL particles, and genome-wide association studies have linked both transporters to HDL cholesterol levels in humans $(5,6)$. Mutations in the human ABCA1 gene result in Tangier disease, and

Authorship note: Yuan Zhang and Sarah R. Breevoort contributed equally to this work. Conflict of interest: David J. Mangelsdorf has ownership in stock of Exelixis Inc. Citation for this article: J Clin Invest. 2012;122(5):1688-1699. doi:10.1172/JCI59817.
Tangier patients characteristically present with little or no HDL and massive accumulation of cholesterol in macrophages found lodged in lymph tissue, and they exhibit an increased risk for atherosclerosis (7-9). The accumulation of oxidized and other modified forms of cholesterol by macrophages present in blood vessel walls is a critical event in the pathogenesis of atherosclerosis (10), and the ability of LXR agonists to enhance macrophage cholesterol efflux has stimulated great interest in the therapeutic potential of these compounds (11). Activation of LXRs also regulates expression of ABCG5 and ABCG8, two half-transporters that dimerize to create an additional cholesterol transporter $(12,13)$. Expression of ABCG5/ABCG8 is largely restricted to the liver and intestine (14), where these proteins function to promote the excretion of cholesterol (liver) and limit cholesterol absorption (intestine). Thus, by mobilizing cholesterol from the periphery, promoting hepatic excretion, and limiting absorption, activation of LXRs results in a net loss of cholesterol. This process of trafficking cholesterol to HDL and ultimately out of the body has been termed reverse cholesterol transport $(\mathrm{RCT})(8,9)$. Importantly, LXR agonists decrease atherosclerosis in animal models, and it has been suggested that enhanced RCT plays an important role in this activity (15-18).

In spite of many potential benefits in cholesterol metabolism, enthusiasm for the therapeutic value of LXR agonists has been tempered by the observation that LXR activation stimulates hepatic lipogenesis by increasing expression of SREBP-1c, a master transcriptional regulator of fatty acid and triglyceride synthesis $(19,20)$. Along with Srebp1c, LXRs regulate either directly or indirectly the genes encoding a number of other proteins involved in fatty acid synthesis $(21,22)$, 
A

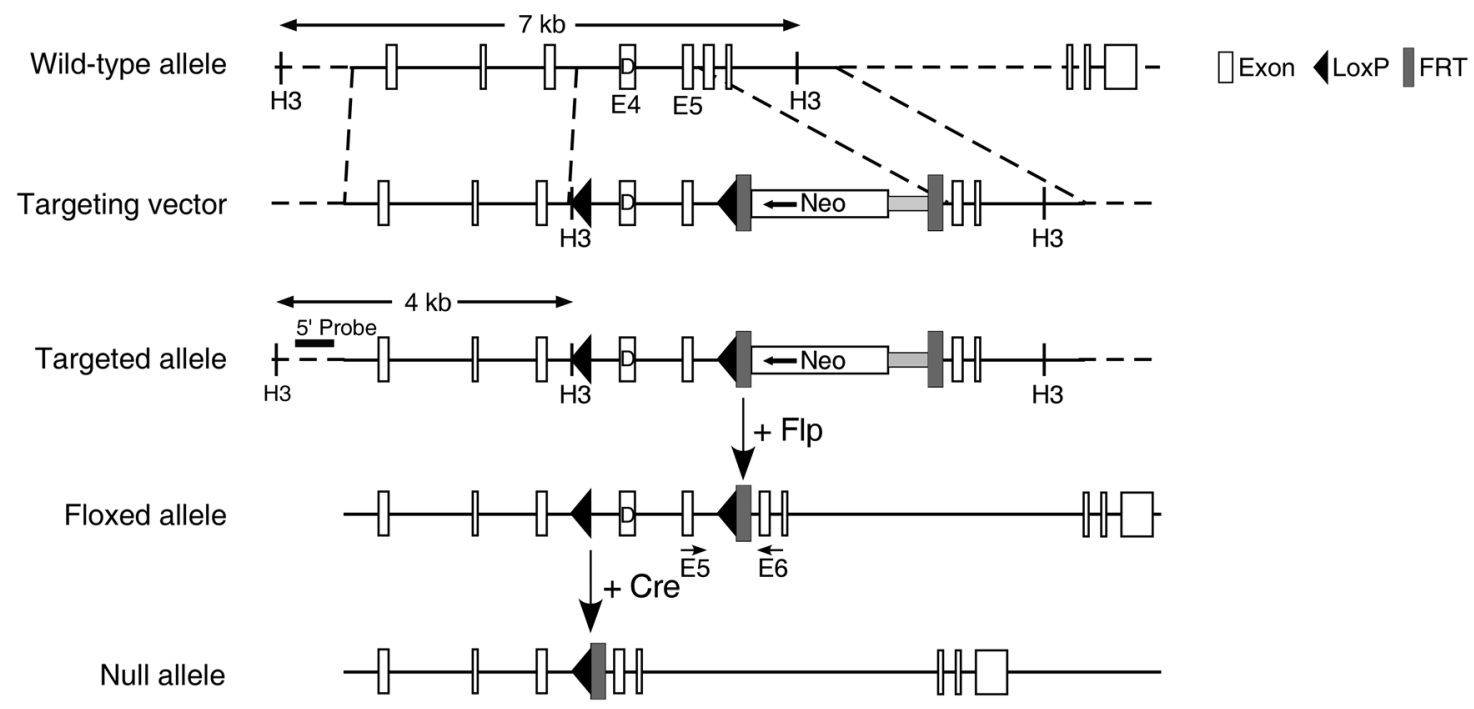

B $\quad \operatorname{LXR} \alpha+/++/+\quad \mathrm{fl} /++\mathrm{fl} /+\mathrm{fl} / \mathrm{fl} \mathrm{fl} / \mathrm{fl}$

Cre $-1-+1-+1-\quad-1-$
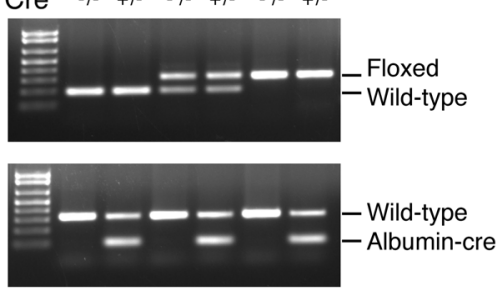

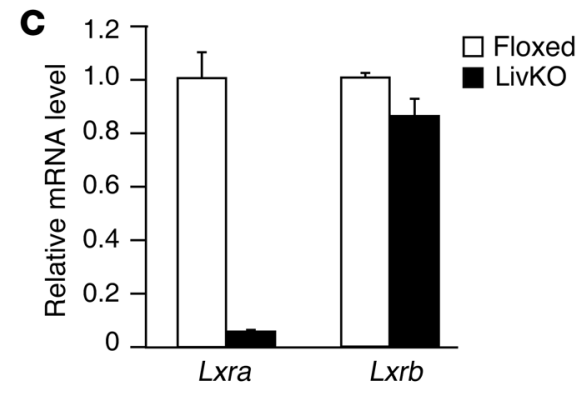

D

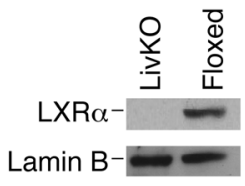

Figure 1

Liver-specific deletion of $L X R \alpha$. (A) Schematic description of the targeting strategy to disrupt hepatic $L X R \alpha$ expression. (B) PCR analysis of the Lxra gene from mice that are wild-type (+/+), Cre (+/-: albumin-cre), flox/+, flox/+: albumin-cre, flox/flox (Flox), and flox/flox: albumin-cre (LivKO). PCR primers for detecting floxed allele are located in exon 5 (E5) and E6 as indicated in A. (C) RT-qPCR analysis of $L x r a$ and $L x r b$ gene expression in liver from floxed and LivKO mice. Data are mean \pm SEM. (D) Western blot analysis of LXR $\alpha$ protein levels using liver nuclear extracts from C57BL/6 floxed and LivKO mice. Nuclear membrane protein lamin B was used as a loading control.

and treatment with LXR agonists can result in dramatic increases in hepatic and plasma triglycerides $(19,20)$. Additionally, at least one class of synthetic LXR ligands has been shown to elevate plasma LDL cholesterol levels in non-human primates (23). Genetic studies have defined the LXR $\alpha$ subtype as the major regulator of hepatic lipogenesis in response to LXR agonists $(15,24)$. The simple idea of creating LXR $\beta$-specific ligands to bypass the undesirable effects on lipogenesis, however, has been challenging because the ligand-binding pockets of the two LXR subtypes differ by only a single amino acid (25, 26). Studies in LDL receptor and Apoe-knockout mice have also demonstrated that it is the LXR $\alpha$ subtype that plays the dominant role in limiting diet-induced cardiovascular disease $(15,27)$. These observations have led to the suggestion that LXR agonists that bypass the liver, or even function as antagonists in the liver, would have ideal therapeutic profiles $(11,28,29)$. To address the therapeutic potential of liver LXR activity, we used gene targeting technology to create a conditional LXR-knockout mouse line by selectively deleting LXR $\alpha$ in hepatocytes. Characterization of these animals demonstrated the essential, physiologic importance of hepatic LXR $\alpha$ to whole body cholesterol homeostasis while at the same time revealing the pharmacologic utility of bypassing hepatic LXR activity as a therapeutic strategy for treating cardiovascular disease.

\section{Results}

Generation of liver-specific $L X R \alpha$-knockout mice. The floxed allele of LXR $\alpha$ was obtained by inserting two loxP sites flanking exons 4 and 5, which encode the DNA binding domain and hinge region of the LXR $\alpha$ (Figure 1A). Homologous recombination was screened by Southern blot analysis with the targeted allele yielding a 4$\mathrm{kb}$ band in addition to a 7-kb wild-type band, and the neomycin resistance cassette was removed by subsequent expression of Flip recombinase. Positive ES clones were injected into blastocysts, and the resulting chimeras were screened for germline transmission. Progeny carrying the floxed allele ( $L x \mathrm{ra}^{\mathrm{fl} / \mathrm{fl}}$ albumin-CRE- ${ }^{-}$, referred to as floxed) were crossed with albumin-Cre mice to generate a hepatocyte-specific knockout of LXR $\alpha\left(L x r a^{f l / f l}\right.$ albumin-CRE ${ }^{+}$, referred to as LivKO) (Figure 1B). Lxra mRNA levels were reduced more than $95 \%$ in livers from hepatocyte-specific knockout mice, while the expression of Lxrb did not change (Figure 1C). No change in Lxra or Lxrb mRNA was detected in any other tissue (Supplemental Figure 1; supplemental material available online with this article; doi:10.1172/JCI59817DS1). LXR $\alpha$ protein was undetectable in liver nuclear extracts of LivKO mice (Figure 1D). Feeding LivKO mice a diet with $2 \%$ cholesterol for 30 days resulted in significant cholesterol accumulation in the liver and an increased 


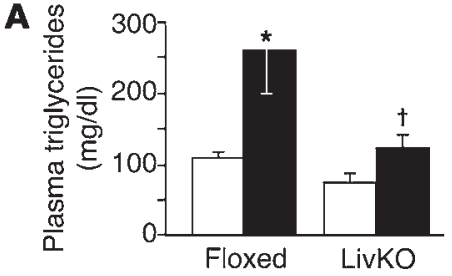

B $\frac{9}{9}$

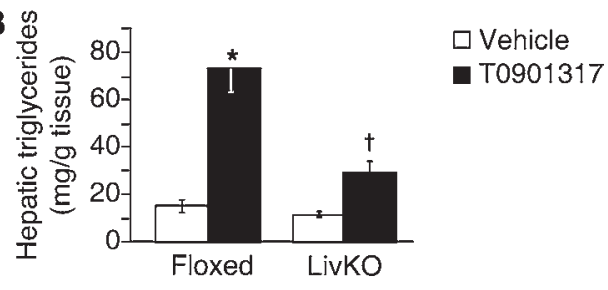

C
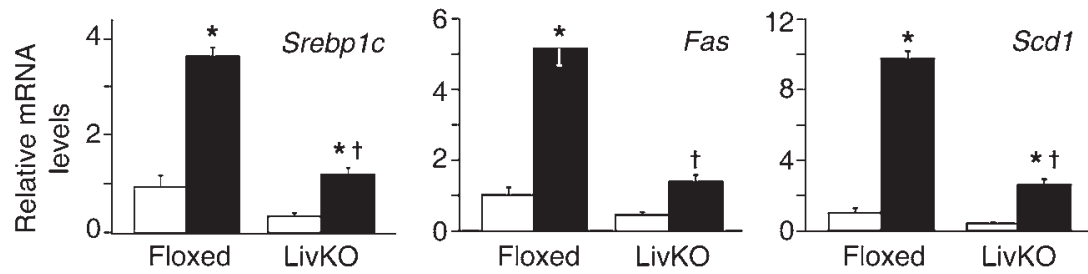

\section{Figure 2}

Hepatic $L X R \alpha$ is required for agonist-dependent increases in triglycerides. Floxed and LivKO female mice 4-6 months of age $(n=5-6)$ were fed a chow diet containing vehicle or T0901317 (40 mpk) for 8 days. (A) Plasma and (B) hepatic triglycerides at days 2 and 8 , respectively, were determined. (C) Liver gene expression was measured using RT-qPCR. Data are mean \pm SEM. ${ }^{*} P \leq 0.05$ between vehicle- and T0901317-treated animals of the same genotype; ${ }^{\top} P \leq 0.05$ between floxed and LivKO mice with the same treatment. liver to body weight ratio (Supplemental Figure 2), essentially recapitulating the phenotype seen with the global LXR $\alpha$ knockout under the same conditions (22).

Regulation of lipid metabolism by hepatic LXR $\alpha$. Treatment with LXR agonists has been shown to increase triglyceride levels, promote cholesterol excretion, and elevate plasma HDL cholesterol (2). To determine the contribution of hepatic LXR $\alpha$ to the biological response to LXR agonists, we fed LivKO and floxed control mice a normal chow diet in the absence or presence of the LXR agonist T0901317. Although T0901317 has been shown to bind to other nuclear receptors as well as LXR (30-33), we chose to use this agonist because it is potent and accumulates to high concentrations in mouse liver, thus allowing maximum activation of hepatic LXR activity. Importantly, the effects of T0901317 on lipid metabolism and atherosclerosis have previously been shown in several studies to be LXR dependent $(15,17,19,20)$. After 2 days of agonist treatment, an increase in plasma triglycerides was observed in control mice that was significantly attenuated in the absence of hepatic LXR $\alpha$ (Figure 2A). The agonist-dependent increase in plasma triglycerides was largely resolved by day 8 (Supplemental Figure 3) - an observation consistent with published data (34) - most likely resulting from the increase in lipoprotein lipase in the liver (35). Nevertheless, hepatic triglycerides remained elevated at day 8 in control agonist-treated mice but were significantly reduced in LivKO mice (Figure 2B). Analysis of hepatic gene expression at day 8 indicated that the agonist-dependent increase in Srebp1c, as well as fatty acid synthase (Fas) and stearoyl CoA desaturase 1 (Scd1), was reduced in LivKO mice (Figure 2C). Interestingly there was a slight but significant increase in Srebp1c in agonist-treated LivKO mice. This residual activity could be due to hepatic LXR $\beta$ activity and/or LXR activity in cells other than hepatocytes, such as Kupffer cells. Previous studies with global knockouts demonstrated that LXR $\alpha$ is a major regulator of hepatic SREBP-1c expression and triglyceride levels $(15,24)$. Taken together, the lipid measurements and gene expression analysis of LivKO mice support this conclusion and further indicate that hepatic LXR $\alpha$ activity is responsible for most of the lipogenic activity of LXR agonists.

Regulation of $A b c g 5$ and $A b c g 8$ in the liver and intestine has been proposed to account for the ability of LXR agonists to stimulate the biliary secretion of cholesterol and decrease intestinal absorption, resulting in increased neutral sterols in the feces $(12,36,37)$. Recent studies, however, have described a biliary-independent trans-intesti- nal pathway for cholesterol excretion that can be stimulated by LXR activity (38-40). As shown in Figure 3, A-C, the ability of T0901317 to increase biliary cholesterol was abolished, and fecal sterols were decreased $85 \%$ in LivKO mice. The loss of agonist activity observed in LivKO mice coincided with a failure to increase $A b c g 5$ and $A b c g 8$ in the liver (Figure 3D). Induction of $A b c g 5$ and $A b c g 8$ in the intestine, however, was not affected (Figure 3E). Therefore, under the experimental conditions used in this study, hepatic LXR $\alpha$ activity was required for the majority of the LXR agonist-dependent increase in cholesterol excretion. These results also support the notion that hepatic, and not intestinal, ABCG5/G8 is required for LXR-dependent effects on cholesterol excretion.

The regulation of bile acid synthesis by LXR has also been suggested to contribute to the ability of LXR agonists to dispose of cholesterol $(22,41)$. As expected, the ability of T0901317 to increase expression of Cyp7a1, which encodes the rate-limiting enzyme in the conversion of cholesterol to bile acids (cholesterol $7 \alpha$-hydroxylase), was absent in LivKO mice (Figure 4A). Nevertheless, no effect of T0901317 treatment on fecal or biliary bile acids or on the bile acid pool size was detected in floxed or LivKO mice (Figure 4, C and D, and Supplemental Figure 4). These results support previous studies showing that LXR synthetic agonists have no effect on fecal bile salt excretion despite the increase in Cyp7a1 expression, which has been suggested to be due to the fast reabsorption and recycling of bile acids (36-38). Along with regulation of Cyp7a1, treatment of control animals with T0901317 resulted in decreased expression of Cyp8b1, the gene encoding sterol $12 \alpha$-hydroxylase, and this effect of agonist was lost in LivKO mice (Figure 4B). Sterol $12 \alpha$-hydroxylase sits at a branch point in the bile acid synthetic pathway, and its enzymatic activity is required for the synthesis of cholic acid (CA). The parallel arm in the pathway leads to synthesis of muricholic acid (MCA) in mice and chenodeoxycholic acid (CDCA) in humans (42). Consistent with the gene expression data, treatment with T0901317 decreased the ratio of CA to MCA in control mice but not in LivKO animals (Figure 4E). Importantly, individual bile acids differ in their ability to promote intestinal cholesterol absorption, and MCA, among all bile acids tested, promotes the lowest amount of cholesterol absorption, while CA promotes the greatest amount $(43,44)$. Thus, the agonist-dependent change in bile acid composition should contribute to the ability of LXR ligands to reduce intestinal cholesterol absorption. Consistent with this hypothesis, the ability of T0901317 to decrease fractional cholesterol absorption 

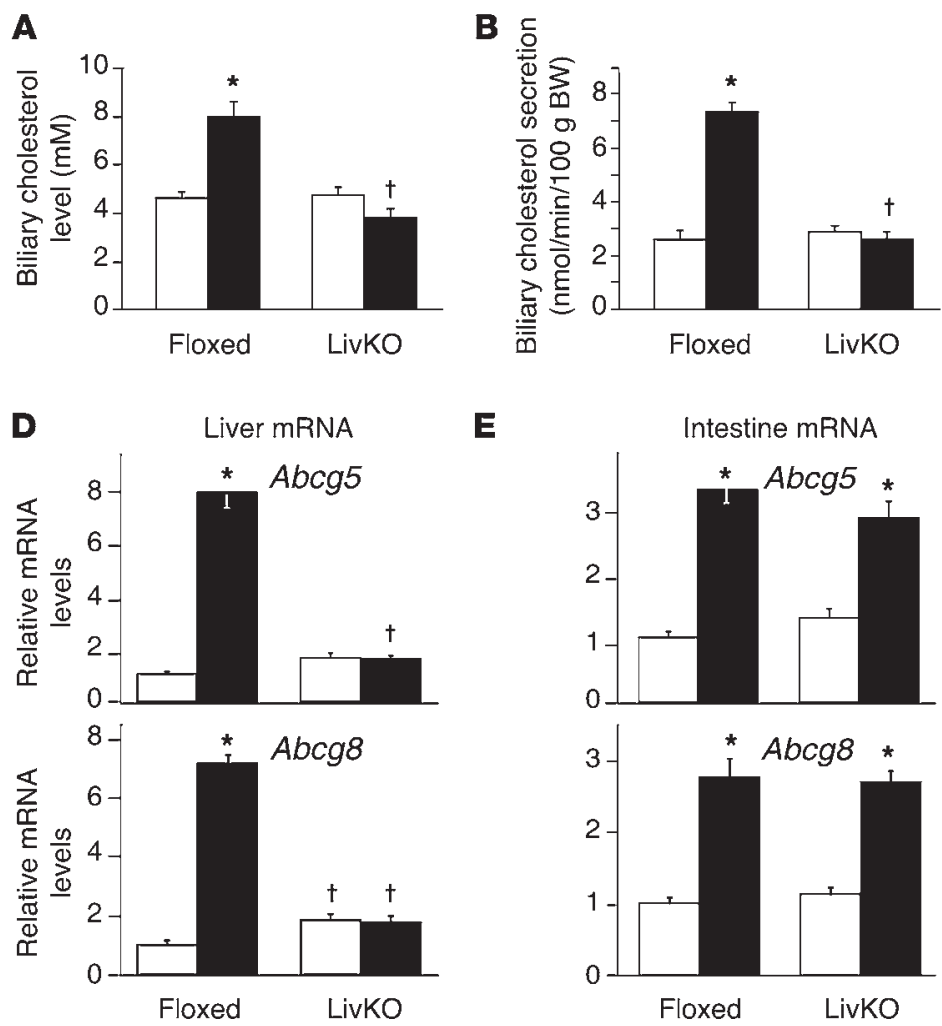

$\mathbf{E}$

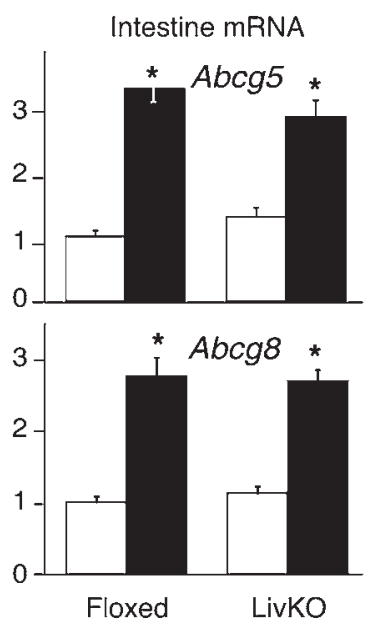

\section{C}

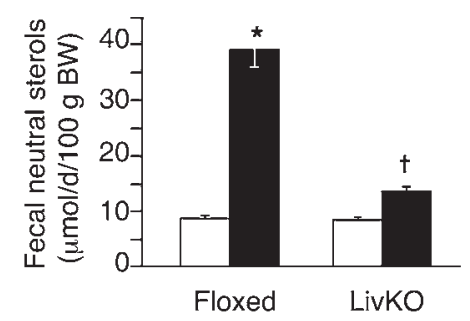

$\square$ Vehicle
$\square$ To901317

Figure 3

Hepatic LXR $\alpha$ is required for agonist-dependent increases in biliary and fecal cholesterol excretion. Floxed and LivKO female mice 5-6 months of age were fed a chow diet containing vehicle or T0901317 (40 mpk) for 3 days. (A) Gallbladder bile was collected and used to determine biliary cholesterol levels $(n=5-6)$. (B) Mice were anesthetized, the common bile duct was cannulated, and bile flow was collected for 30 minutes. Biliary cholesterol and secretion rate were determined $(n=3-6)$. (C-E) Floxed and LivKO female mice 4-6 months of age $(n=5-6)$ were fed a chow diet containing vehicle or T0901317 (40 mpk) for 8 days. (C) Fecal neutral sterol secretion was determined by gas chromatography. Liver (D) and intestine (E) gene expression was assayed by quantitative real-time PCR. Data are mean \pm SEM. ${ }^{*} P \leq 0.05$ between vehicle- and T0901317-treated animals of the same genotype; ${ }^{\dagger} P \leq 0.05$ between floxed and LivKO mice with the same treatment.

was also significantly attenuated in LivKO mice (Figure 4F). The failure to increase biliary cholesterol excretion (Figure 3B) - which can dilute the radiolabeled cholesterol used to quantitate absorption by transporting large amounts of unlabeled cholesterol into the intestinal lumen - most likely also contributes to the agonistdependent reduction in fractional cholesterol absorption. Biliary phospholipid levels were reduced by T0901317, indicating that biliary cholesterol and phospholipid excretion can be uncoupled by LXR agonist activity (Supplemental Figure 4, C and D).

Hepatic LXR $\alpha$ is not required for agonist-dependent HDL cholesterol regulation. The liver is considered the major site of HDL production $(45,46)$, and treatment of chow-fed mice with LXR agonists is known to increase HDL cholesterol levels $(19,20)$. After treatment with T0901317 for 8 days, a significant increase in plasma cholesterol was observed in both LivKO and control mice, although the levels in T0901317-treated LivKO mice were approximately $15 \%$ lower than in control animals (Figure 5A). Fractionation of lipoprotein particles by fast protein liquid chromatography (FPLC) indicated that the increase primarily resided in the HDL fraction (Figure 5, C and D). Thus, LXR $\alpha$ activity in the liver is not required in order for LXR agonists to increase HDL cholesterol levels in the plasma. The increase in fractions 24-30 detected with both groups of treated animals has been observed in several other studies and was shown to be due to the presence of large HDL particles $(13,34,38)$. Interestingly, this shoulder was smaller in T0901317-treated LivKO mice compared with controls (Figure 5, C and D), suggesting that hepatic $\operatorname{LXR} \alpha$ can play a role in modulating HDL size. Consistent with these results, expression of several apolipoproteins was altered in LivKO mice (Supplemental Figure 5). ABCA1 is required for the biogenesis of HDL, and studies with ABCA1-knockout mice indicate that both the liver and intestine contribute to HDL production $(45,46)$. Induction of Abca1 mRNA by T0901317 was almost completely abrogated in the livers of LivKO mice, while the intestinal Abca1 levels were not different from those in control mice (Figure 5, E and F). The strong LXR agonist-dependent induction of Abca1 in the intestine suggests that this organ serves as a major site for LXR-dependent HDL cholesterol increases, a conclusion supported by earlier studies using tissue-specific knockouts of $A b c a 1$ and intestinespecific overexpression of $\operatorname{LXR} \alpha(47,48)$.

Hepatic cholesterol levels were increased in vehicle-treated LivKO mice (Figure 5B), even though we did not detect significant differences in biliary cholesterol or bile acid secretion (Figure 3 and Supplemental Figure 4). The mRNA levels of HMG-CoA synthase, however, were paradoxically increased in these animals, raising the possibility that hepatic cholesterol synthesis is elevated in LivKO 

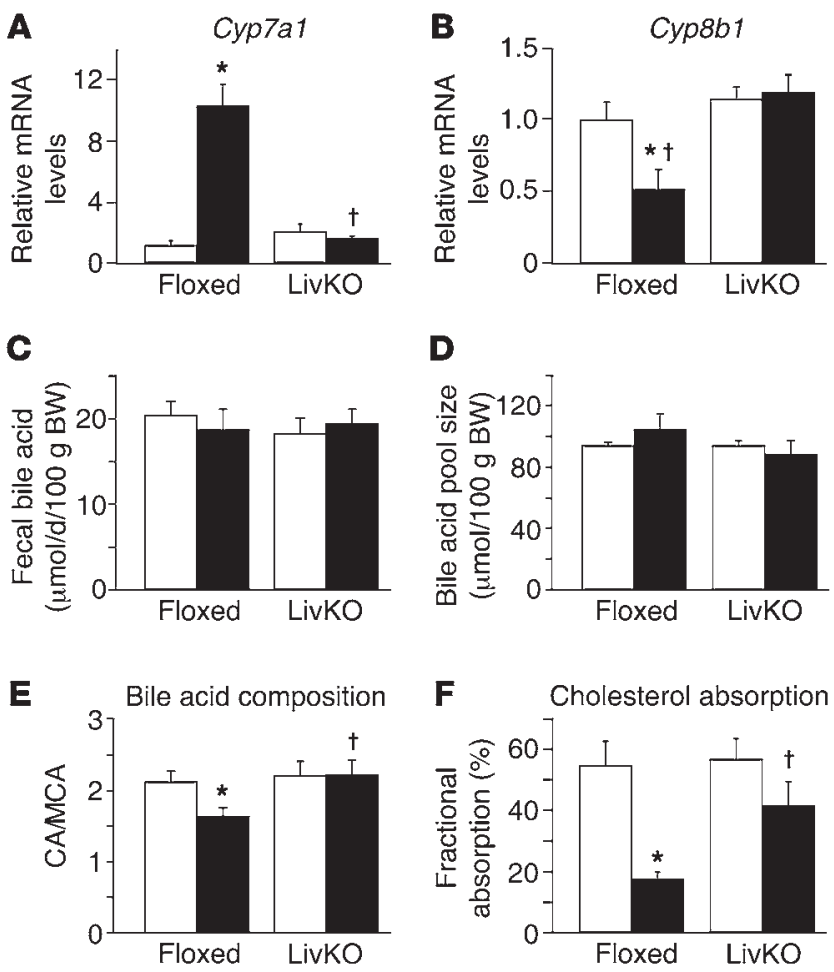

\section{Figure 4}

Hepatic $L X R \alpha$ is required for agonist-dependent changes in bile acid composition and fractional cholesterol absorption. Floxed and LivKO female mice 4-6 months of age $(n=5-6)$ were fed a chow diet containing vehicle or T0901317 (40 mpk) for 8 days. Hepatic mRNA expression of Cyp7a1 (A) and Cyp8b1 (B) was assayed by quantitative real-time PCR. (C) Fecal bile acid secretion was determined by a $3 \alpha$-hydroxysteroid dehydrogenase assay. (D) Bile acid pool size was determined by liquid chromatography/mass spectrometry. (E) Bile acid composition was measured, and the ratio of CA to MCA was calculated. (F) Cholesterol absorption efficiency was determined by dual-isotope method. Data are mean \pm SEM. ${ }^{*} P \leq 0.05$ between vehicle- and T0901317-treated animals of the same genotype; ${ }^{\dagger} P \leq 0.05$ between floxed and LivKO mice with the same treatment. mice on a chow diet (Supplemental Figure 5). A similar increase in the expression of cholesterol biosynthetic genes has been observed in the livers of the complete LXR $\alpha$ knockout (22), although the mechanism that accounts for the misregulation of HMG-CoA synthase in the absence of LXR remains to be determined. Treatment with T0901317 significantly reduced hepatic cholesterol in both floxed and LivKO mice, a result consistent with recent studies demonstrating that increased LXR activity in the intestine can reduce hepatic cholesterol (48).

Deletion of hepatic LXR $\alpha$ increases atherosclerosis. LXR agonists decrease atherosclerosis in animal models of cardiovascular disease (15-18), and global deletion of LXR $\alpha$ increases atherosclerosis in either LDL receptor $(L d l r)$-knockout or Apoe-knockout genetic backgrounds $(15,27)$. Using a series of bone marrow transplantations, we previously demonstrated that cells derived from the hematopoietic system constitute an important site of LXR-dependent anti-atherogenic activity $(15,17)$. These studies, however, also indicated important anti-atherogenic functions for LXR $\alpha$ in a site (or sites) that is not derived from bone marrow cells (15). To determine the impact of liver LXR $\alpha$ activity on atherosclerosis, we introduced the liver-specific knockout into the $L d l r^{-/}$background. The resulting double knockouts $\left(L d l r^{-/-} L x r a a^{f / f l} /\right.$ albumin-CRE ${ }^{+}$; i.e., $L d l r^{-/-} /$LivKO) and littermate controls ( $L d l r^{-/-} L x \mathrm{raf}^{f / f l} /$ albumin-CRE ; i.e., $L d l r^{-1-} /$ floxed) were placed on a high-fat/high-cholesterol Western diet (21\% fat, $0.15 \%$ cholesterol) for 20 weeks in the absence or presence of T0901317. By 4 weeks on the diet, $L d l r^{-/} /$LivKO animals had reduced plasma triglycerides and cholesterol compared with controls, and the effect of T0901317 on plasma lipid levels was lost in the Ldlr-/-/LivKO mice (Supplemental Figure 6, A-D). Consistent with other studies in hyperlipidemic mouse models $(15-18,27)$, treatment with LXR agonist had little or no effect on HDL cholesterol levels in either $L d l r^{-/} /$floxed or $L d l r^{-/-} /$LivKO animals (Supplemental Figure 6, E and F). As expected, hepatic cholesterol was substantially increased in $\mathrm{Ldlr}^{-/} / \mathrm{LivKO}$ animals at the conclusion of the experiment (Supplemental Figure 7).

When atherosclerosis was quantitated by en face analysis of dissected aortas or by serial sections of the aortic root, a significant increase in lesion area was detected in $\mathrm{Ldlr}^{-1} /$ LivKO mice compared with controls (Figure 6 and Supplemental Figure 8). Immunostaining with the macrophage-specific antibody MOMA2 indicated increased macrophage content in $\mathrm{Ldlr}^{-/-} / \mathrm{LivKO}$ root sections. Collagen staining, a measure of plaque stability, was roughly similar (Supplemental Figure 9). A similar increase in atherosclerosis was also observed in $\mathrm{Ldl}^{-/} /$LivKO mice after 10 weeks on a Western diet (Supplemental Figure 10). Thus, LXR $\alpha$ activity in the liver plays an essential role in limiting cardiovascular disease in the background of the Ldlr-null animal. Importantly, T0901317 was still able to significantly reduce atherosclerosis in Ldlr/-/LivKO mice (Figure 6 and Supplemental Figures 8 and 10), indicating that liver $\operatorname{LXR} \alpha$ activity is not required for the pharmacological anti-atherogenic activity of LXR agonists. The magnitude of the agonist-dependent decrease in $\mathrm{Ldlr}^{-/} / \mathrm{LivKO}$ mice was similar to that observed in $\mathrm{Ldlr}^{-/} /$floxed controls (30\%-40\%), suggesting that the full therapeutic effect of LXR agonists can be manifested in the absence of liver LXR $\alpha$.

Lipoprotein particle number, size, and function in LivKO mice. We noted that $\mathrm{Ldlr}^{/-} /$LivKO mice had relatively high plasma cholesterol levels, while their plasma triglyceride levels were approximately 5 times lower than those in $\mathrm{Ldlr}^{-/}$-/floxed controls (Supplemental Figure 6, A-D). This large difference in plasma triglycerides suggested the possibility that the number and/or size of the lipoprotein particles produced in $\mathrm{Ldl} \mathrm{r}^{/-} /$LivKO mice may be altered in a way that influences atherogenesis. To address this possibility, we used NMR spectroscopy to examine lipoprotein particle number and size (49). The high triglyceride levels in T0901317-treated Ldlr/-/floxed mice precluded 


\section{A}

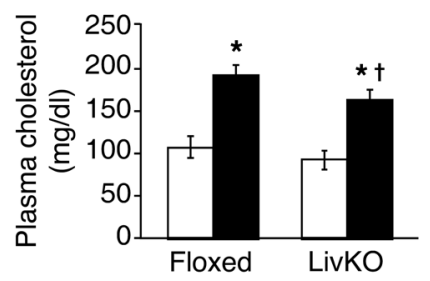

B

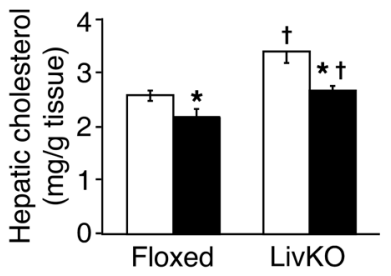

C

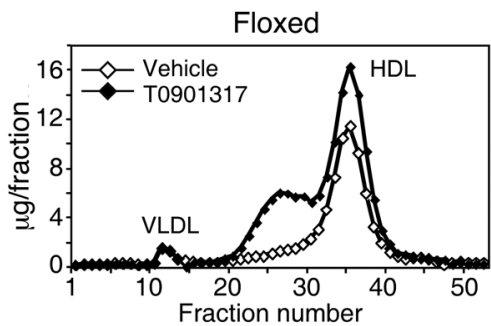

D

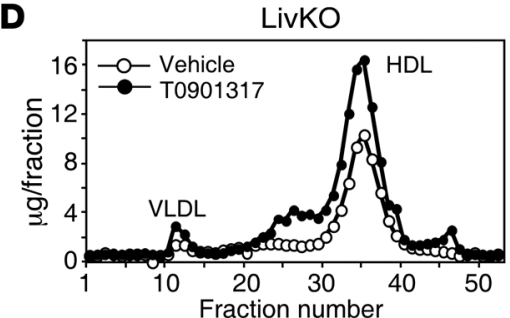

$\mathbf{E}$

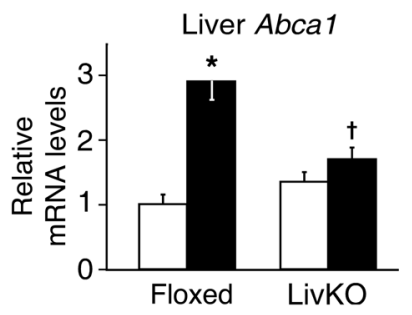

$\mathbf{F}$

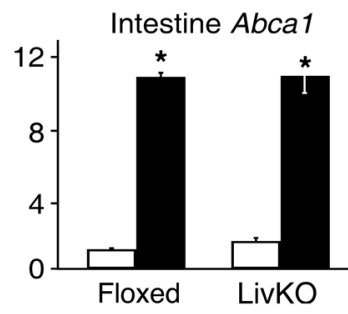

$\square$ Vehicle

- T0901317

Figure 5

LXR agonists increase HDL cholesterol in the absence of hepatic LXR $\alpha$. Floxed and LivKO female mice $4-6$ months of age $(n=5-6)$ were fed a chow diet containing vehicle or T0901317 (40 mpk) for 8 days. (A) Plasma total cholesterol and $(\mathbf{B})$ hepatic cholesterol were determined as described in Methods. Plasma from floxed (C) and LivKO (D) mice treated with or without T0901317 was pooled and subjected to FPLC for lipoprotein analysis. Cholesterol content of each fraction was assayed enzymatically. Gene expression from liver (E) and small intestine (F) harvested at completion of the experiment was assayed by quantitative real-time PCR. Data are mean \pm SEM. ${ }^{*} P \leq 0.05$ between vehicle- and T0901317-treated animals of the same genotype; ${ }^{\dagger} P \leq 0.05$ between floxed and LivKO mice with the same treatment. analysis of the effect of agonist treatment on particle number and size by NMR, so we restricted this analysis to vehicle-treated animals that had been on a Western diet for 10 weeks. As expected, the number of VLDL particles was decreased in $\mathrm{Ldl}^{/ /} /$LivKO mice (Table 1), consistent with an important role for hepatic LXR $\alpha$ in triglyceride synthesis. In contrast, while $L d l r^{-/} /$floxed and $L d l r^{-/} /$LivKO animals had similar numbers of LDL particles, there was a dramatic change in particle size, with almost $50 \%$ of the $\mathrm{Ldlr}^{-/} / \mathrm{LivKO}$ particles having diameters less than $21 \mathrm{~nm}$ (Table 1 , small LDL). We note that the high percentage of relatively large LDL particles (diameter, $\geq 23 \mathrm{~nm}$ ) measured in $L d l r^{-/} /$floxed mice is consistent with previous studies in hyperlipidemic mice (50). Although there is a clear difference in size between LDL particles of the two strains, we did not detect a difference in cholesterol accumulation when bone marrow-derived macrophages were cultured in vitro in the presence of plasma or FPLC-purified apoB-containing lipoproteins from $\mathrm{Ldl} /$-/ / floxed or Ldlr/-/LivKO animals (data not shown).

Both FPLC (Supplemental Figure 6) and NMR (Table 1) indicate that there is no difference in HDL cholesterol levels between the $L d r^{-/} /$floxed and $L d l r^{-/} /$LivKO animals. The $L d l r^{-/} /$LivKO mice, however, did have a $30 \%$ decrease in total HDL particle number that was largely the result of a decrease in small HDL (particles with diameters smaller than $8.2 \mathrm{~nm}$; Table 1). To examine if the change in particle number and size influences HDL function, we performed in vitro cholesterol efflux assays using ${ }^{3} \mathrm{H}$-cholesterolloaded RAW264.7 cells. Cholesterol efflux was significantly reduced when Ldlr/-/LivKO plasma (Figure 7A) or FPLC-purified HDL (Figure $7 \mathrm{~B}$ ) was used as the source of cholesterol acceptors. The analysis of lipoprotein number, size, and function identifies hepatic LXR $\alpha$ as an important regulator of lipoprotein metabolism and suggests that alterations in LDL and/or HDL function may contribute to the increased atherosclerosis observed in $\mathrm{Ldll}^{-/} / \mathrm{LivKO}$ mice.
RCT is impaired in LivKO mice. The ability to excrete cholesterol from the liver into the bile is a critical step in the RCT pathway. Our studies in normal lipidemic mice indicate that the ability of LXR agonists to stimulate cholesterol excretion is lost in the absence of hepatic LXR $\alpha$ activity (Figure 3); nevertheless, T0901317 still retains anti-atherogenic activity (Figure 6 and Supplemental Figure 10). To examine RCT under hyperlipidemic conditions, we injected mouse J774 cells loaded with ${ }^{3} \mathrm{H}$-cholesterol and acetylated LDL in vitro into the peritoneal cavity of $\mathrm{Ldlr}^{-/} /$floxed and $L d l r^{-/} /$LivKO mice that been on a Western diet for 9 weeks in the absence or presence of T0901317. The amount of ${ }^{3} \mathrm{H}$ in the plasma, liver, and feces was determined 48 hours later (Figure 8 and Supplemental Figure 11). The ability of T0901317 to increase the fecal excretion of macrophage-derived sterols was largely impaired in $\mathrm{Ldlr}^{-/-} /$LivKO mice (Figure 8A and Supplemental Figure 11, $\mathrm{A}-\mathrm{C})$. Concurrently there was an increase in ${ }^{3} \mathrm{H}$-sterol in the livers of $\mathrm{Ldlr}^{-/} / \mathrm{LivKO}$ mice (Figure 8B), indicating that hepatic LXR $\alpha$ is needed for agonist-dependent fecal excretion of macrophagederived cholesterol. The ability of LXR agonists to increase the efflux of macrophage-derived ${ }^{3} \mathrm{H}$-cholesterol into the plasma is thought to result from agonists acting on macrophage LXRs to enhance ABCA1- and ABCG1-dependent cholesterol efflux (51, 52). Consistent with other studies (51-53), treatment of $\mathrm{Ldlr}^{-1-}$ floxed mice with T0901317 produced a time-dependent increase in the level of ${ }^{3} \mathrm{H}$-cholesterol in the plasma (Figure 8C). Interestingly, the level of ${ }^{3} \mathrm{H}$-cholesterol in the plasma of $\mathrm{Ldlr}^{-/-} / \mathrm{LivKO}$ mice was decreased relative to that of vehicle-treated $L d l r^{-/} /$floxed controls, and treatment with T0901317 had no effect (Figure 8C). For all 4 groups, FPLC analysis indicated that the distribution of ${ }^{3} \mathrm{H}$ tracer in the plasma exactly coincided with the distribution of bulk, unlabeled cholesterol (data not shown). To determine whether the decrease in plasma ${ }^{3} \mathrm{H}$-cholesterol levels observed in 

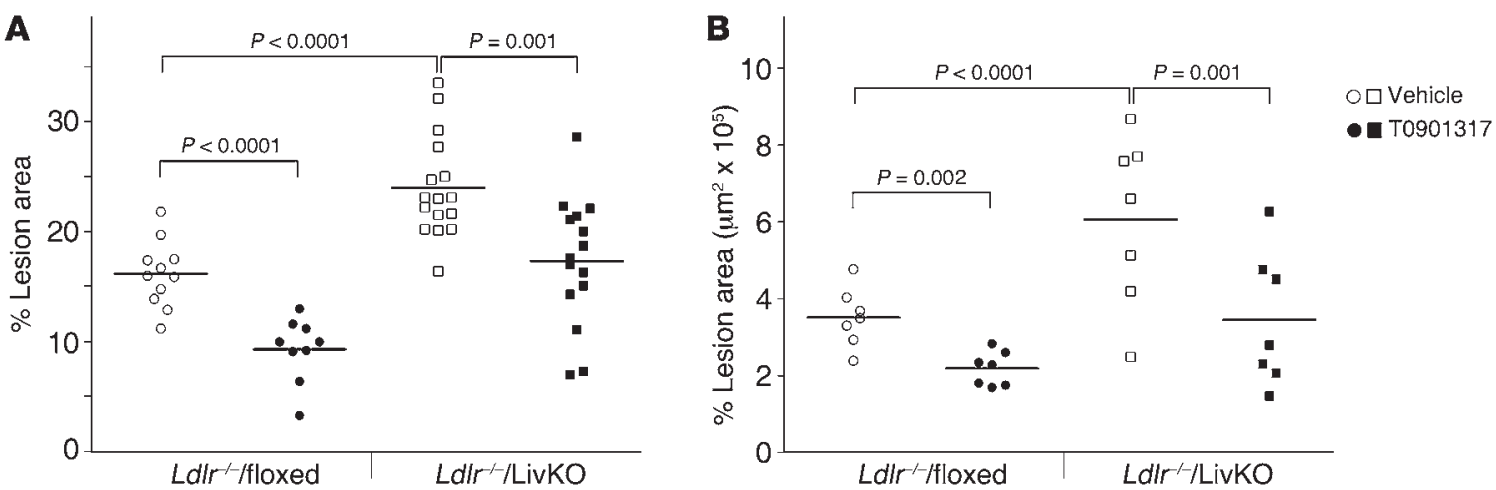

\section{Figure 6}

Atherosclerosis in LdIr/-/LivKO mice. Mice were fed a Western diet with or without $0.01 \%$ T0901317 for 20 weeks, and atherosclerosis was quantitated. (A) En face analysis of the aorta was carried out as described in Methods. Ldlr-//floxed (vehicle: $n=11,6$ male, 5 female; T0901317: $n=$ 9, 5 male, 4 female). Ldlr $r^{-/} /$LivKO (vehicle: $n=16,8$ male, 8 female; T0901317: $n=15,8$ male, 7 female). (B) Lesion area was measured in serial sections of the aortic root ( $n=7 /$ group; 4 male, 3 female). Each data point represents an individual animal. The horizontal lines denote the mean of each group. There were no significant differences between sexes within any of the groups.

Ldlr-/LivKO animals resulted from impaired LXR transcriptional activity in macrophages, we recovered the J774 cells from the peritoneal cavity 48 hours after injection and quantitated $A b c a 1$ mRNA levels in RNA isolated from these cells. As shown in Figure 8D, agonist treatment produced a similar increase in $A b c a 1$ mRNA in cells recovered from $L d l r^{-/-} /$floxed and $L d l r^{-/} /$LivKO animals. Additionally, a similar increase in Abca1 mRNA was observed in RNA isolated from whole blood taken from animals on a Western diet in the absence or presence of T0901317 for 10 weeks (Supplemental Figure 11D). The gene expression analysis suggests that the failure of LXR agonist to increase the efflux of macrophage-derived cholesterol into the plasma of $L d l r^{-1-} /$ LivKO mice does not arise from a defect in macrophage LXR activity.

\section{Discussion}

LXRs function throughout the body to control cholesterol transport, catabolism, and excretion (11). This report describes what we believe to be the first conditional LXR-knockout mouse model constructed by selective elimination of the LXR $\alpha$ subtype in hepatocytes (LivKO mice). When challenged with a $2 \%$ cholesterol diet, LivKO mice accumulated increased amounts of cholesterol in liver resulting from failure to induce hepatic cholesterol excretion and catabolism, highlighting the importance of liver LXR $\alpha$ activity to whole body cholesterol homeostasis. Similarly, the ability of synthetic LXR agonists to stimulate biliary cholesterol excretion, inhibit fractional cholesterol absorption, and increase the output of neutral sterols in the feces was largely compromised in LivKO mice. Several recent studies have described a trans-intestinal pathway for cholesterol excretion that bypasses biliary excretion but nevertheless can be stimulated by LXR activation (38-40). Our studies suggest that such a biliaryindependent pathway makes only a minor contribution to LXR agonist-dependent cholesterol excretion.

Early studies with synthetic LXR agonists described increases in plasma triglycerides and plasma HDL cholesterol as two pharmacological responses to LXR activation $(19,20)$. Analysis of LivKO mice indicates that these responses originate from unique sites. The LXR agonist-dependent increases in triglycerides were of hepatic origin and resulted from regulation of the genes encod- ing SREBP-1c and other enzymes involved in fatty acid and triglyceride synthesis. In contrast, hepatic deletion of LXR $\alpha$ had little effect on the ability of LXR agonists to elevate HDL cholesterol. Both the liver and intestine have been shown to contribute to the production of HDL $(45-47,54)$, and while the LXR agonist-dependent induction of $A b c a 1$, encoding a protein required for HDL biogenesis, was impaired in liver, induction of $A b c a 1$ in the intestine was unchanged. The tissue-specific expression of Abca1 observed in LivKO mice suggests that LXR activation in the intestine is sufficient to regulate HDL cholesterol levels. Consistent with our conclusion that intestinal LXR activity is primarily responsible for elevating HDL cholesterol, previous studies indicate that expression of ABCA1 in the intestine is required for LXR agonist-dependent cholesterol increases (47) and that transgenic overexpression of a constitutively active LXR $\alpha$ (VP16LXR $\alpha$ ) in the intestine increases HDL (48). HDL cholesterol levels inversely correlate with cardiovascular disease risk, and the ability of LXR agonists to increase HDL cholesterol initially stimulated great interest in the therapeutic potential of such com-

\section{Table 1}

Lipoprotein particle size and number

\begin{tabular}{|c|c|c|}
\hline Particles & $L d / r^{-/ / f l o x e d}$ & LdIr/-/LivKO \\
\hline VLDL, total, nmol/l & $727 \pm 128$ & $341 \pm 130^{A}$ \\
\hline Large VLDL (>60 nm), nmol/l & $15.7 \pm 4.5$ & $4.4 \pm 1.3^{\mathrm{A}}$ \\
\hline Medium VLDL (35-60 nm), nmol// & $179 \pm 103$ & $117 \pm 81$ \\
\hline Small VLDL $(27-35 \mathrm{~nm}), \mathrm{nmol} / \mathrm{l}$ & $533 \pm 67$ & $220 \pm 98 \mathrm{~A}$ \\
\hline LDL, total, nmol/l & $1238 \pm 257$ & $1440 \pm 463$ \\
\hline$-27 \mathrm{~nm}), \mathrm{nmol} / \mathrm{l}$ & $1181 \pm 246$ & $685 \pm 212^{A}$ \\
\hline Large LDL (21.2-23 nm), nmol/l & 0 & 0 \\
\hline Small LDL (18-21.2 nm), nmol/l & 0 & $742 \pm 364^{A}$ \\
\hline HDL particles, total $\mu \mathrm{mol} / \mathrm{l}$ & $34.6 \pm 9.6$ & $24 \pm 2.7^{A}$ \\
\hline Large HDL (8.8-13 nm), $\mu \mathrm{mol} / \mathrm{l}$ & $14.2 \pm 6.4$ & $16.7 \pm 4.6$ \\
\hline Medium HDL (8.2-8.2 nm), $\mu \mathrm{mol} / \mathrm{l}$ & 0 & 0 \\
\hline Small HDL (7.3-8.2 nm), $\mu \mathrm{mol} / \mathrm{l}$ & $18 \pm 3.2$ & $7.3 \pm 4.7$ \\
\hline $\mathrm{HDL}$ cholesterol, mg/dl & $50.6 \pm 25.8$ & $60.3 \pm 17$ \\
\hline
\end{tabular}

AP $\leq 0.05$ between floxed and LivKO mice ( $n=6 /$ group). IDL, intermediate-density lipoprotein. 
A

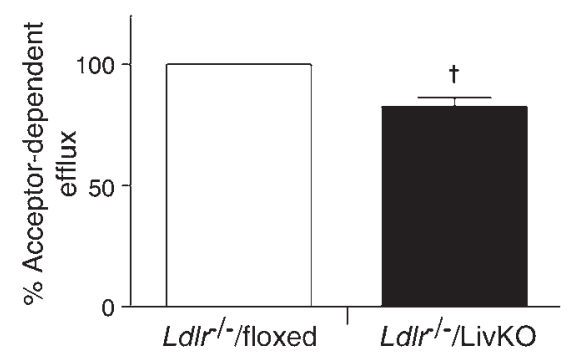

B

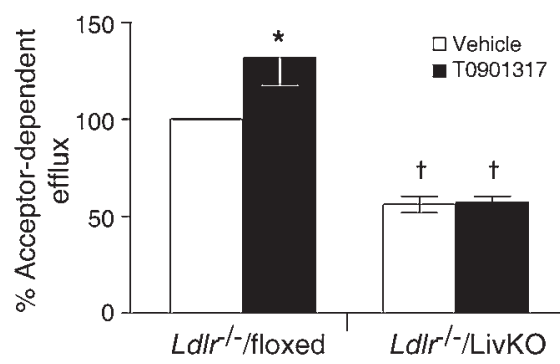

Figure 7

Cholesterol efflux in RAW264.7 cells. ${ }^{3} \mathrm{H}$-Cholesterol-loaded RAW264.7 cells were cultured with (A) $0.03 \%$ plasma or (B) FPLC-purified HDL isolated from mice fed a Western diet with or without $0.01 \%$ T0901317 for 9 weeks, and cholesterol efflux was determined as described in Methods. Efflux to plasma or HDL isolated from Ldl/ $r^{-/ / f l o x e d ~ m i c e ~ f e d ~ a ~ W e s t e r n ~ d i e t ~ w i t h o u t ~ T 0901317 ~ w a s ~ s e t ~ a s ~} 100 \%$. Data are the average of 2 independent experiments and expressed as mean \pm SEM. Data from the experiments with FPLC-purified HDL were normalized to the amount of apoAl added. ${ }^{*} P \leq 0.05$ between vehicle- and T0901317-treated animals of the same genotype; ${ }^{\dagger} P \leq 0.05$ between floxed and LivKO mice.

pounds (11). The concurrent increase in lipogenesis, however, has dampened the enthusiasm for LXR agonists and slowed the progression of therapeutic molecules into the clinic. Analysis of LivKO mice demonstrates that the lipogenic and HDL pathways are tissue specific and suggests that LXR ligands that specifically target the intestine, for instance by limiting systemic absorption or by rapid first-pass clearance, could have therapeutic value.

In mouse models of cardiovascular disease, treatment with LXR agonists decreases atherosclerosis. However, in these hyperlipidemic models LXR agonists have little or no effect on HDL cholesterol levels, and this has led to the conclusion that the anti-atherogenic activity originates from increased macrophage cholesterol efflux and/or limiting inflammation in immune cells in atherosclerotic plaque (15-18). Indeed, selective deletion of LXR $\alpha$ in hematopoietic cells increased atherosclerosis in the $L d l r^{-/}$background, although the increase was not as great as that measured in $\mathrm{Ldlr}^{-/} \mathrm{Lxra}^{-/-}$global knockout mice $(15,17,55)$. We now demonstrate that atherosclerosis was substantially increased when LXR $\alpha$ was selectively eliminated in hepatocytes, identifying the liver as a critical site of LXR $\alpha$-dependent anti-atherogenic activity. Our studies suggest that hepatic LXR $\alpha$ modulates lipoprotein particle number, size, and function in a manner that influences atherogenicity. In particular, the ability of HDL to accept cholesterol from macrophages was impaired in $\mathrm{Ldlr}^{-1-} / \mathrm{LivKO}$ mice. In addition we note that the decrease in cholesterol acceptor ability observed with HDL in Ldlr-//LivKO mice correlates with the loss of the large HDL fraction observed in normal lipidemic LivKO mice. These observations suggest that pharmacological strategies utilizing small molecules that inhibit hepatic LXR $\alpha$ activity to reduce lipogenesis may actually increase cardiovascular disease and should be explored with caution. Future studies that examine the effect of hepatic LXR activity on lipoprotein function in the presence of the cholesteryl ester transfer protein (CETP), a lipoprotein particle-remodeling enzyme expressed in humans but not mice (56), will be useful in this regard.

Despite the increased atherosclerosis observed in $\mathrm{Ldlr}^{-1-} /$ LivKO mice, treatment with T0901317 was still an effective preventive therapy, indicating that extrahepatic LXR activity can also be anti-atherogenic. Our in vivo RCT analysis further suggests that the ability of LXR agonists to stimulate the RCT pathway is substantially compromised in the absence of hepatic LXR $\alpha$ and is thus not necessary for the athero-preventive activ- ity of LXR agonists. The efficacy of agonist treatment in LivKO mice therefore raises questions regarding the potential mechanisms and sites of action for the pharmacological activity of LXR agonists. In contrast to our findings in liver, using bone marrow transplantations we previously showed that LXR activity in hematopoietic cells is necessary for the anti-atherogenic activity of T0901317 (17). A number of additional functions for LXRs in immune cells including the control of inflammation (2), endoplasmic reticulum stress (57), macrophage egress $(58)$, and monocyte proliferation $(59,60)$ could underlie the anti-atherogenic activity of LXR ligands. Finally, recent studies indicate that intestine-specific activation of LXRs using pharmacological or transgenic approaches can increase RCT and may beneficially impact the treatment of atherosclerosis $(48,61)$. The failure of LXR agonist treatment to increase the appearance of macrophage-derived cholesterol in the plasma of $\mathrm{Ldlr}^{-1-} / \mathrm{LivKO}$ mice during the in vivo RCT assay further raises the possibility that impaired LXR activity in the liver can negatively affect macrophage cholesterol efflux in the periphery. The appearance of macrophage-derived ${ }^{3} \mathrm{H}$-cholesterol in the plasma during the in vivo RCT assay, however, may not simply reflect the rate of the macrophage cholesterol efflux. The reentry of ${ }^{3} \mathrm{H}$-cholesterol into the plasma compartment after uptake by the liver and/or intestine may also contribute to this measurement. Therefore, we cannot rule out the possibility that LXR agonists do in fact promote macrophage cholesterol efflux in $L d l^{-/-} /$LivKO mice and that this activity is anti-atherogenic even when hepatic cholesterol excretion to the bile is inhibited. In summary, our characterization of LivKO mice demonstrates that while endogenous hepatic LXR $\alpha$ activity is essential for maintaining normal lipid and sterol homeostasis, pharmacologic strategies that bypass LXR activation in liver may still be of therapeutic benefit.

\section{Methods}

Reagents. LXR agonist T0901317 was purchased from Cayman Chemical.

Generation of floxed LXR $\alpha$ mice. High-fidelity PCR amplification of $129 \mathrm{SvEv}$ genomic DNA was used to generate an approximately $3.5-\mathrm{kb}-$ long arm including exons 1,2 , and 3 ; an approximately 0.8 -kb targeting arm including exons 4 and 5; and an approximately 1.5 -kb short arm including exons 6 and 7 . These fragments were assembled in the pMC1neo PolyA vector (Stratagene) containing a neomycin resistance cassette and loxP and FRT sites. KpnI-linearized DNA was electroporated into $129 \mathrm{SvEv}$ - 
A

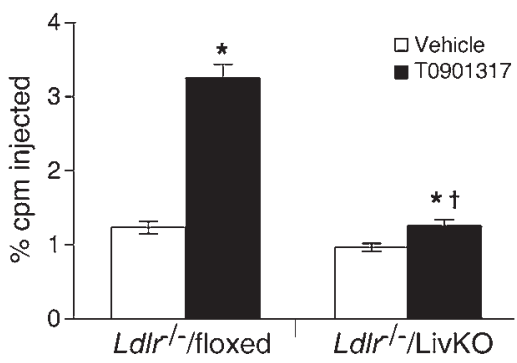

C

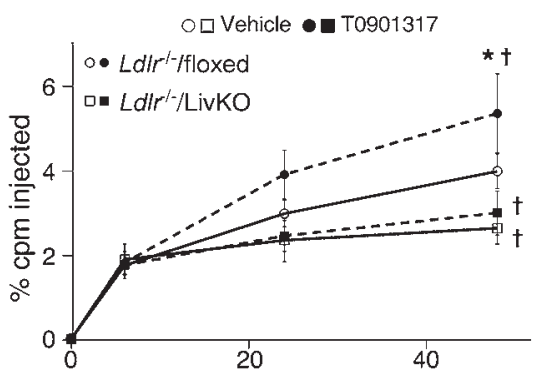

B

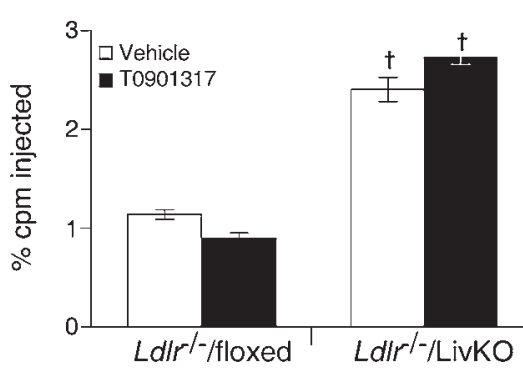

D J774.A1 Abca1 mRNA

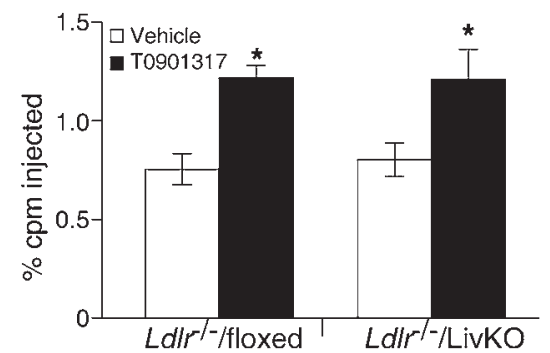

Figure 8

In vivo $\mathrm{RCT} .{ }^{3} \mathrm{H}$-cholesterol and acetylated LDL-loaded J774 macrophages were injected into mice fed a Western diet with or without $0.01 \%$ T0901317 for 10 weeks ( $n=6$ / group), and the amount of ${ }^{3} \mathrm{H}$ tracer in feces (A), liver (B), and plasma (C) was determined as described in Methods. (D) Total RNA was isolated from recovered $\mathrm{J} 774$ macrophages, and the mRNA levels of Abca1 were measured by quantitative real-time PCR. Data are mean \pm SEM. ${ }^{*} P \leq 0.05$ between vehicleand T0901317-treated animals of the same genotype; ${ }^{\dagger} P \leq 0.05$ between floxed and LivKO mice with the same treatment. derived ES cells. ES cells were screened for targeted recombination by PCR and Southern blot analysis using a 5' probe and genomic DNA digested with HindIII. One positive ES cell clone was electroporated with plasmid expressing Flipase (a gift from Susan Dymecki, Department of Genetics, Harvard Medical School, Boston, Massachusetts, USA) to remove the neo cassette, and the resultant recombination was screened by PCR. Two independently derived ES cell clones were injected into C57BL/6J blastocysts to produce chimeric mice that transmitted the modified Lxra locus. The genotype of the progenies of the mice carrying the floxed allele was analyzed by PCR using a forward primer (LXR $\alpha$ E5), 5'-GTGTAACAGGCGCTCCTTCT-3'; and reverse primer (LXR $\alpha$ E6), 5'-GGGCAAAGCGCTGTTGTCG-3'. The amplification was carried out in a thermocycler using the following conditions: $94^{\circ} \mathrm{C} 30$ seconds, $58^{\circ} \mathrm{C} 45$ seconds, $72^{\circ} \mathrm{C}$ 1 minute for 35 cycles. The wild-type allele amplified a 200-bp fragment; the floxed allele amplified a 300-bp fragment.

Animal experiments. Floxed LXR $\alpha$ mice were bred with C57BL/6J albumin-Cre transgenic mice (The Jackson Laboratory) to generate liver-specific knockout animals (LivKO). Albumin-Cre ${ }^{-}$floxed littermates were used as their control. All animals were housed in a temperature-controlled environment with 12-hour light/12-hour dark cycles. Age-matched mice had free access to water and were fed standard rodent chow (TD 7001, Harlan Teklad), a high-cholesterol diet (TD 86295, Harlan Teklad) (22), or standard rodent chow in powder mixed with LXR agonist T0901317 (40 mg per $\mathrm{kg}$ of body weight) or vehicle (1\% Tween- 80 in $10 \%$ methylcellulose). At the end of the experiment, mice were anesthetized and exsanguinated. Blood was collected in EDTA-coated tubes, and tissues were harvested for analyses.

$L d l r^{-/} L x r a^{f l / f l} /$ albumin-CRE- $\left(L d l r^{-/} /\right.$floxed) and $L d l r /-L x r a f / f l /$ albumin$\mathrm{CRE}^{+}\left(\mathrm{Ldll}^{-/} / \mathrm{LivKO}\right)$ mice were created by mating $L x \mathrm{raf} / \mathrm{fl} / \mathrm{albumin}-\mathrm{CRE}^{+}$ mice with $L d l r^{-/-}$mice (male B6.129S7-Ldl $r^{\text {tm } 1 \text { Her }} / \mathrm{J}$ ) purchased from The Jackson Laboratory. Mice were fed standard chow ad libitum until the beginning of the study. To examine atherosclerosis in $\mathrm{Ldl} / \mathrm{r} /$ /floxed and Ldlr ${ }^{-1 / L i v K O}$ mice, we placed 8- to 9-week-old animals on a Western diet (21\% fat wt/wt, $0.15 \%$ cholesterol wt/wt; Test Diet 57BD) with or without $0.01 \%$ T0901317 added to the diet for 10 or 20 weeks.
Plasma analyses. Blood was collected into EDTA-coated tubes (Starstedt). Plasma was separated by centrifugation and assayed for total cholesterol (Thermo Scientific) and triglycerides (Thermo Scientific). Plasma lipoprotein levels were analyzed by FPLC using Superose HR6 columns, followed by enzymatic assays for total cholesterol and triglycerides. Elevated plasma triglyceride levels in samples from $L d l r^{-/-} L x r^{f l} / f l /$ albumin-CRE- mice treated with T0901317 resulted in a substantial amount of non-HDL cholesterol aggregating when samples were centrifuged to pellet particulate matter prior to loading the FPLC column. Therefore, the non-HDL cholesterol levels measured by FPLC for $L d l r^{-/} L x_{r a f l / f l} /$ albumin-CRE- animals treated with T0901317 most likely represent an underestimate.

Liver cholesterol and triglycerides. Liver lipids were extracted in Folch solution (chloroform/methanol, 2:1, v/v) (62). Liver samples (0.1 g) were homogenized in $4 \mathrm{ml}$ Folch. Extracts were then washed once with $1 \mathrm{ml}$ of $50 \mathrm{mM} \mathrm{NaCl}$ and twice with $1 \mathrm{ml}$ of $0.36 \mathrm{M} \mathrm{CaCl}_{2} /$ methanol. The organic phase was separated and brought up to $5 \mathrm{ml}$ with chloroform. Fifty microliters of each sample and $10 \mu \mathrm{l}$ of standards were mixed with $10 \mu \mathrm{l}$ of $50 \%$ Triton X-114 in chloroform (v/v). Samples were air dried and then subjected to colorimetric enzymatic assays for total cholesterol (Thermo Scientific) or triglycerides (Thermo Scientific)

Fractional cholesterol absorption. Intestinal cholesterol absorption was determined by a fecal dual-isotope ratio method (63). Individually housed mice were on a chow diet mixed with either vehicle or T0901317 for 8 days. On the fifth day, mice were dosed intragastrically with a mixture of $2 \mu \mathrm{Ci}$ $\left[5,6-{ }^{3} \mathrm{H}\right]$ sitostanol and $1 \mu \mathrm{Ci}\left[4-{ }^{14} \mathrm{C}\right]$ cholesterol in $100 \mu \mathrm{l}$ medium-chain triglyceride oil. Mice were returned to fresh cages, and feces were collected for 3 days. One gram of dried and ground feces from each animal was extracted with chloroform/methanol $(2: 1, \mathrm{v} / \mathrm{v})$, and the content of ${ }^{14} \mathrm{C}$ and ${ }^{3} \mathrm{H}$ in each sample was determined. The percentage of cholesterol absorption was determined as described previously (63).

Fecal bile acids and neutral sterols. Mice were individually housed and fed a chow diet containing either vehicle or T0901317 (40 milligrams per kilogram [mpk]) for 8 days. Feces were collected over the last 4 days and were dried, weighed, and ground to a powder. An aliquot of $0.5 \mathrm{~g}$ of the feces 
was used to determine the total bile acid content using a $3 \alpha$-hydroxysteroid dehydrogenase assay (64). A second aliquot of $0.5 \mathrm{~g}$ of the feces was extracted, and the neutral sterol content (cholesterol, coprostanol, and $5 \alpha$ cholestan-3-ol) was quantitated by gas chromatography (65). 5-Cholestene was added as an internal control.

Bile acid pool size and composition. Mice were fed chow diet containing either vehicle or T0901317 (40 mpk) for 8 days and then fasted for 4 hours before sacrifice. Gallbladder, liver, and intestine were removed and placed into $50 \mathrm{ml}$ EtOH. The solution was spiked with $50 \mu \mathrm{g}$ CDCA-D 4 (C/D/N Isotopes), and tissues were extracted as described previously (66). Bile acids were quantified by liquid chromatography/mass spectrometry (Agilent Technologies). Quantification was performed based on peak areas using external calibration curves of standards prepared in methanol. CDCA-D $\mathrm{D}_{4}$ was used to calculate the recovery of bile acids after extraction relative to a blank control.

Bile flow and biliary lipid composition. Mice were fed a chow diet containing either vehicle or T0901317 (40 mpk) for 3 days and then anesthetized by intraperitoneal injection of tribromoethanol (Avertin, $250 \mathrm{mg} / \mathrm{kg}$ ) after a 4-hour fast. Gallbladders were removed, and the common bile duct was cannulated with a polyethylene-10 catheter. Hepatic bile was collected for 30 minutes. Bile flow was determined gravimetrically assuming a density of $1 \mathrm{~g} / \mathrm{ml}$ for bile. Concentrations of cholesterol (Thermo Scientific), bile salts (Diagnostic Chemicals), and phospholipids (Waco) from gallbladder and hepatic bile were determined enzymatically.

Western blotting. Liver nuclear proteins were extracted as described previously (19). Nuclear proteins $(100 \mu \mathrm{g})$ were resolved on a $10 \%$ SDS-polyacrylamide gel and electrotransferred to a PVDF membrane (Amersham). The membrane was then hybridized with mouse anti-human LXR $\alpha$ antibody (PPMX) at a 1:1500 dilution, followed by secondary antibody incubation at a 1:5,000 dilution. $\mathrm{LXR} \alpha$ protein was detected by chemiluminescence (ECL kit, Amersham).

Quantitative real-time PCR analysis. Total RNA was extracted from liver and small intestine using RNA STAT-60 (Tel-Test Inc.). RNA was treated with DNase I and then reverse transcribed into cDNA with random hexamers using the SuperScript II First-Strand Synthesis System (Invitrogen). Primers for each gene were designed using Primer Express Software (Applied Biosystems) and were validated as previously described (67). Quantitative real-time PCR (RT-qPCR) reactions contained $25 \mathrm{ng}$ of cDNA, $150 \mathrm{nM}$ of each primer, and $5 \mu$ l of SYBR GreenER PCR Master Mix (Invitrogen) and were carried out in triplicate using an Applied Biosystems Prism 7900HT instrument. Relative mRNA levels were calculated using the comparative Ct method and normalized to cyclophilin.

Quantitation of atherosclerosis. Atherosclerosis, macrophage levels, and collagen staining in root sections and en face preparations were quantitated as previously described $(17,55,68)$.

NMR lipoprotein measurements. $L d l r^{-/} /$floxed and $L d l / /-/$ LivKO mice were fed a Western diet with or without $0.01 \%$ T0901317 ( $n=6 /$ group). After 10 weeks, mice were euthanized, and blood was collected into EDTA-coated tubes and centrifuged at $900 \mathrm{~g}$ for 15 minutes at $4^{\circ} \mathrm{C}$ to prepare plasma. Lipoprotein particle concentrations and sizes in plasma samples were analyzed by NMR spectroscopy by LipoScience Inc. (49).

Cholesterol efflux experiments. RAW264.7 cells were plated in 96-well plates $\left(2 \times 10^{5}\right.$ cells/well $)$ and 24 hours later were labeled with $1 \mu \mathrm{Ci} / \mathrm{ml}^{3} \mathrm{H}$-cholesterol (PerkinElmer) in DMEM plus 1\% FBS media for 18-24 hours. Radiolabeled cells were washed with prewarmed PBS and incubated for 6 hours with serum-free media containing $0.03 \%$ pooled plasma $(n=6$ animals/group) or 25\% FPLC-purified HDL as cholesterol acceptors. Following incubation with acceptors, media was collected and radioactivity was measured by liquid scintillation counting. Cells were washed with PBS and lysed in $100 \mu \mathrm{l}$ of $0.2 \mathrm{~N} \mathrm{NaOH}$, and radioactivity in cell lysates was quantitated by liquid scintillation counting. Cholesterol efflux was expressed as percentage of $\mathrm{cpm}$ in the medium divided by the total counts $\left(c p m_{\text {media }} /\left[c p m_{\text {media }}+c p m_{\text {cell }}\right]\right)$. Acceptor-dependent efflux was determined by subtracting the efflux of vehicle cells cultured without acceptor. apoAI $(10 \mu \mathrm{g} / \mathrm{ml})$ or HDL $(15 \mu \mathrm{g} / \mathrm{ml})$ was included as positive control. Data are expressed as mean \pm SEM of at least 8 wells/treatment. Experiments were repeated 3 times with similar results. For experiments with FPLC-purified HDL, data were normalized to the amount of apoAI added as determined by Western blotting.

Macrophage RCT experiments. In vivo RCT experiments were carried out as described by Naik et al. (51) in $L d l r^{-/} /$floxed and $L d l r^{-/} /$LivKO mice fed a Western diet with or without $0.01 \%$ T0901317 for 9 weeks. Briefly, J774 cells were loaded with $25 \mu \mathrm{g} / \mathrm{ml}$ acetylated LDL and $5 \mu \mathrm{Ci} / \mathrm{ml}^{3} \mathrm{H}$ cholesterol for 48 hours in vitro. Cholesterol-loaded cells were injected into the peritoneal cavity of mice $\left(4.5 \times 10^{6} \mathrm{cells} /\right.$ mouse, $3 \times 10^{6} \mathrm{cpm}$, $n=6 /$ group), which were housed individually for the duration of the experiment. Blood was collected at 6,24, and 48 hours after injection, and the ${ }^{3} \mathrm{H}$-choelsterol in triplicate plasma samples was determined by scintillation counting. Levels of ${ }^{3} \mathrm{H}$ tracer in the liver, total fecal ${ }^{3} \mathrm{H}$ sterols, fecal ${ }^{3} \mathrm{H}$-choelsterol, and fecal ${ }^{3} \mathrm{H}$-bile acids were measured as described by Naik et al. (51).

To measure gene expression in J774 cells recovered from the peritoneal cavity of mice, cells were collected 48 hours after injection, lysed with PureZOL (Bio-Rad), and extracted with chloroform, and total RNA was isolated from the aqueous layer using an RNeasy kit (QIAGEN). RNA was treated with DNase I and then reverse transcribed into CDNA with random hexamers using a High-Capacity cDNA Reverse Transcription kit (Applied Biosystems). RT-qPCR reactions contained $20 \mathrm{ng}$ of cDNA, $385 \mathrm{nM}$ of each primer, and $8 \mu \mathrm{l}$ of iQ SYBR Green Supermix (Bio-Rad) and were carried out in triplicate using a Bio-Rad MyiQ instrument. Relative mRNA levels were calculated using the comparative $\mathrm{Ct}$ method and normalized to cyclophilin. Primers for mouse ABCA1 were: forward, 5'-GCTCTCAGGTGGGATGCAG-3'; reverse, 5'-GGCTCGTCCAGAATGACAAC-3'. Primers for mouse cyclophilin were: forward, 5'-CGATGACGAGCCCTTGG-3'; reverse, 5'-TCTGCTGTCTTTGGAACTTTGTC-3'.

Gene expression in whole blood. Fresh whole blood (approximately $350 \mu \mathrm{l}$ ) was collected in heparinized capillary tubes and transferred to Eppendorf tubes, and cells were lysed with $0.7 \mathrm{ml}$ PureZOL (Bio-Rad). Following lysis, $150 \mu \mathrm{l}$ chloroform was added, and total RNA was isolated from the aqueous layer using and RNeasy kit (QIAGEN). Abca1 mRNA levels were measured and normalized to cyclophilin as described above.

Statistics. Results were analyzed by 1-way ANOVA or 2-tailed Student's unpaired $t$ test, using GraphPad Prism (GraphPad Software); $P$ values of 0.05 or less were considered significant.

Study approval. All animal experiments were approved by the Institutional Animal Care and Research Advisory Committee of the University of Texas Southwestern Medical Center and the University of Virginia.

\section{Acknowledgments}

We thank Calli Merkel for technical help, Robert Hammer for generation of the LXR $\alpha$ floxed mice, Fang Xu and Jonathan Cohen for neutral sterol analysis, and Irena Ignatova-Torodova for help with the RCT assays. This research was supported by the Howard Hughes Medical Institute (to D.J. Mangelsdorf), NIH grants U19DK62434 (to D.J. Mangelsdorf) and 1R01HL096864-01A1 (to I.G. Schulman), and the Robert A. Welch Foundation (I-1275 to D.J. Mangelsdorf and I-1588 to S.A. Kliewer).

Received for publication July 5, 2011, and accepted in revised form February 22, 2012. 
Address correspondence to: Ira Schulman, Department of Pharmacology, University of Virginia, 1300 Jefferson Park Ave., P.O. Box 800735, Charlottesville, Virginia 22908, USA. Phone 434.924.5682; Fax: 434.982.3878; E-mail: igs4c@virginia.edu. Or to: David Mangelsdorf, Department of Pharmacology, Howard Hughes Medical Institute, University of Texas Southwestern
Medical Center, 5323 Harry Hines Blvd., Dallas, Texas 75390, USA. Phone: 214.645.5957; Fax: 214.645.5969; E-mail: davo. mango@utsouthwestern.edu.

Mingui Fu's present address is: Department of Basic Medical Sciences, University of Missouri-Kansas City, Kansas City, Missouri, USA.
1. Steinberg D. The cholesterol controversy is over. Why did it take so long? Circulation. 1989; 80(4):1070-1078.

2. Calkin AC, Tontonoz P. Liver $\mathrm{x}$ receptor signaling pathways and atherosclerosis. Arterioscler Thromb Vasc Biol. 2010;30(8):1513-1518.

3. Chen W, Chen G, Head DL, Mangelsdorf DJ, Russell DW. Enzymatic reduction of oxysterols impairs LXR signaling in cultured cells and the livers of mice. Cell Metab. 2007;5(1):73-79.

4. Janowski BA, Willy PJ, Devi TR, Falck JR, Mangelsdorf DJ. An oxysterol signaling pathway mediated by the nuclear receptor LXR $\alpha$. Nature. 1996;383(6602):728-731.

5. Edmondson AC, et al. Dense genotyping of candidate gene loci identifies variants associated with high-density lipoprotein cholesterol. Circ Cardiovasc Genet. 2011;4(2):145-155.

6. Teslovich TM, et al. Biological, clinical and population relevance of 95 loci for blood lipids. Nature. 2010;466(7307):707-713.

7. Oram JF, Lawn RM. ABCA1. The gatekeeper for eliminating excess tissue cholesterol. J Lipid Res. 2001;42(8):1173-1179.

8. Rader DJ, Alexander ET, Weibel GL, Billheimer J, Rothblat GH. The role of reverse cholesterol transport in animals and humans and relationship to atherosclerosis. J Lipid Res. 2009;(50 suppl):S189-S194.

9. Tall AR, Yvan-Charvet L, Terasaka N, Pagler T, Wang N. HDL, ABC transporters, and cholesterol efflux: implications for the treatment of atherosclerosis. Cell Metab. 2008;7(5):365-375.

10. Glass CK, Witztum JL. Atherosclerosis: the road ahead. Cell. 2001;104(4):503-516.

11. Schulman IG, Heyman RA. LXR as a therapeutic target for atherosclerosis. In: Packard CJ, Rader DJ, eds. Lipids and Atherosclerosis. London, United Kingdom: Taylor \& Francis; 2006:93-100.

12. Repa JJ, Berge KE, Pomajzl C, Richardson JA, Hobbs $\mathrm{H}$, Mangelsdorf DJ. Regulation of ATP-binding cassette sterol transporters ABCG5 and ABCG8 by the liver X receptors alpha and beta. J Biol Chem. 2002;277(21):18793-18800.

13. Yu L, York J, von Bergmann K, Lutjohann D, Cohen JC, Hobbs HH. Stimulation of cholesterol excretion by the liver X receptor agonist requires ATP-binding cassette transporters G5 and G8. J Biol Chem. 2003;278(18):15565-15570

14. Berge KE, et al. Accumulation of dietary cholesterol in sitosterolemia caused by mutations in adjacent $\mathrm{ABC}$ transporters. Science. 2000;290(5497):1771-1775.

15. Bischoff ED, et al. Non-redundant roles for LXRalpha and LXRbeta in atherosclerosis susceptibility in low density lipoprotein receptor knockout mice. J Lipid Res. 2010;51(5):900-906.

16. Joseph SB, et al. Synthetic LXR ligand inhibits the development of atherosclerosis in mice. Proc Natl Acad Sci U S A. 2002;99(11):7604-7609.

17. Levin $N$, et al. Macrophage liver $X$ receptor is required for antiatherogenic activity of LXR agonists. Arterioscler Thromb Vasc Biol. 2005;25(1):135-142.

18. Terasaka N, et al. T-0901317, a synthetic liver $\mathrm{X}$ receptor ligand, inhibits development of atherosclerosis in LDL receptor-deficient mice. FEBS Lett. 2003;536(1-3):6-11.

19. Repa JJ, et al. Regulation of mouse sterol regulatory element-binding protein-1c gene (SREBP-1c) by oxysterol receptors, LXRalpha and LXRbeta. Genes Dev. 2000;14(22):2819-2830.
20. Schultz JR, et al. Role of LXRs in control of lipogenesis. Genes Dev. 2000;14(22):2831-2838.

21. Joseph SB, et al. Direct and indirect mechanisms for regulation of fatty acid synthase gene expression by liver X receptors. J Biol Chem. 2002; 277(13):11019-11025.

22. Peet DJ, et al. Cholesterol and bile acid metabolism are impaired in mice lacking the nuclear oxysterol receptor LXR $\alpha$. Cell. 1998;93(5):693-704.

23. Groot PH, et al. Synthetic LXR agonists increase LDL in CETP species. J Lipid Res. 2005;46(10):2182-2191.

24. Quinet EM, et al. Liver X receptor (LXR)-beta regulation in LXRalpha-deficient mice: implications for therapeutic targeting. Mol Pharmacol. 2006;70(4):1340-1349.

25. Svensson S, et al. Crystal structure of the heterodimeric complex of LXRalpha and RXRbeta ligandbinding domains in a fully agonistic conformation. EMBO J. 2003;22(18):4625-4633.

26. Williams $S$, et al. X-ray crystal structure of the liver $\mathrm{X}$ receptor beta ligand binding domain: regulation by a histidine-tryptophan switch.J Biol Chem. 2003;278(29):27138-27143.

27. Bradley MN, et al. Ligand activation of LXR beta reverses atherosclerosis and cellular cholesterol overload in mice lacking LXR alpha and apoE. J Clin Invest. 2007;117(8):2337-2346.

28. Lund EG, Menke JG, Sparrow CP. Liver X receptor agonists as potential therapeutic agents for dyslipidemia and atherosclerosis. Arterioscler Thromb Vasc Biol. 2003;23(7):1169-1177.

29. Motoshima K, Noguchi-Yachide T, Sugita K, Hashimoto Y, Ishikawa M. Separation of alpha-glucosidase-inhibitory and liver $\mathrm{X}$ receptor-antagonistic activities of phenethylphenyl phthalimide analogs and generation of LXRalpha-selective antagonists. Bioorg Med Chem. 2009;17(14):5001-5014.

30. Houck KA, et al. T0901317 is a dual LXR/FXR agonist. Mol Genet Metab. 2004;83(1-2):184-187.

31. Kumar N, et al. The benzenesulfoamide T0901317 [N-(2,2,2-trifluoroethyl)-N-[4-[2,2,2-trifluoro-1hydroxy-1-(trifluoromethyl)ethyl]phenyl]-benzenesulfonamide] is a novel retinoic acid receptor-related orphan receptor-alpha/gamma inverse agonist. Mol Pharmacol. 2010;77(2):228-236.

32. Mitro N, Vargas L, Romeo R, Koder A, Saez E. T0901317 is a potent PXR ligand: implications for the biology ascribed to LXR. FEBS Lett. 2007;581(9):1721-1726

33. Xue Y, Chao E, Zuercher WJ, Willson TM, Collins JL, Redinbo MR. Crystal structure of the PXRT1317 complex provides a scaffold to examine the potential for receptor antagonism. Bioorg Med Chem. 2007; 15(5):2156-2166.

34. Grefhorst A, et al. Stimulation of lipogenesis by pharmacological activation of the liver $X$ receptor leads to production of large, triglyceride-rich very low density lipoprotein particles. J Biol Chem. 2002;277(37):34182-34190.

35. Zhang Y, Repa JJ, Gauthier K, Mangelsdorf DJ. Regulation of lipoprotein lipase by the oxysterol receptors, LXRalpha and LXRbeta. J Biol Chem. 2001;276(46):43018-43024.

36. Plosch $\mathrm{T}$, et al. Increased hepatobiliary and fecal cholesterol excretion upon activation of the liver $\mathrm{X}$ receptor is independent of ABCA1. J Biol Chem. 2002;277(37):33870-33877.

37. Yu L, et al. Expression of ABCG5 and ABCG8 is required for regulation of biliary cholesterol secre- tion. J Biol Chem. 2005;280(10):8742-8747.

38. Kruit JK, et al. Increased fecal neutral sterol loss upon liver $\mathrm{X}$ receptor activation is independent of biliary sterol secretion in mice. Gastroenterology. 2005;128(1):147-156

39. van der Veen JN, et al. Activation of the liver X receptor stimulates trans-intestinal excretion of plasma cholesterol. J Biol Chem. 2009;284(29):19211-19219.

40. Temel RE, et al. Biliary sterol secretion is not required for macrophage reverse cholesterol transport. Cell Metab. 2010;12(1):96-102.

41. Lehmann JM, et al. Activation of the nuclear receptor LXR by oxysterols defines a new hormone response pathway. J Biol Chem. 1997;272(6):3137-3140.

42. Russell DW. The enzymes, regulation, and genetics of bile acid synthesis. Annu Rev Biochem. 2003;72:137-174.

43. Wang DQ, Lammert F, Cohen DE, Paigen B, Carey MC. Cholic acid aids absorption, biliary secretion, and phase transitions of cholesterol in murine cholelithogenesis. Am J Physiol. 1999;276(3 pt 1):G751-G760.

44. Wang DQ, Tazuma S. Effect of beta-muricholic acid on the prevention and dissolution of cholesterol gallstones in C57L/J mice. J Lipid Res. 2002;43(11):1960-1968.

45. Brunham LR, et al. Intestinal ABCA1 directly contributes to HDL biogenesis in vivo. J Clin Invest. 2006;116(4):1052-1062.

46. Timmins JM, et al. Targeted inactivation of hepatic Abca 1 causes profound hypoalphalipoproteinemia and kidney hypercatabolism of apoA-I.J Clin Invest. 2005;115(5):1333-1342.

47. Brunham LR, Kruit JK, Pape TD, Parks JS, Kuipers F, Hayden MR. Tissue-specific induction of intestinal ABCA1 expression with a liver $\mathrm{X}$ receptor agonist raises plasma HDL cholesterol levels. Circ Res. 2006;99(7):672-674.

48. Lo Sasso G, et al. Intestinal specific LXR activation stimulates reverse cholesterol transport and protects from atherosclerosis. Cell Metab. 2010;12(2):187-193.

49. Jeyarajah EJ, Cromwell WC, Otvos JD. Lipoprotein particle analysis by nuclear magnetic resonance spectroscopy. Clin Lab Med. 2006;26(4):847-870.

50. Hammad SM, Powell-Braxton L, Otvos JD, Eldridge L, Won W, Lyons TJ. Lipoprotein subclass profiles of hyperlipidemic diabetic mice measured by nuclear magnetic resonance spectroscopy. Metabolism. 2003;52(7):916-921.

51. Naik SU, et al. Pharmacological activation of liver $\mathrm{X}$ receptors promotes reverse cholesterol transport in vivo. Circulation. 2006;113(1):90-97.

52. Wang X, et al. Macrophage ABCA1 and ABCG1, but not SR-BI, promote macrophage reverse cholesterol transport in vivo. J Clin Invest. 2007; 117(8):2216-2224.

53. Nijstad N, Gautier T, Briand F, Rader DJ, Tietge UJ. Biliary sterol secretion is required for functional in vivo reverse cholesterol transport in mice. Gastroenterology. 2011;140(3):1043-1051.

54. Singaraja RR, et al. Both hepatic and extrahepatic ABCA1 have discrete and essential functions in the maintenance of plasma high-density lipoprotein cholesterol levels in vivo. Circulation. 2006;114(12):1301-1309.

55. Tangirala RK, et al. Identification of macrophage liver $\mathrm{X}$ receptors as inhibitors of atherosclerosis. Proc Natl Acad Sci U S A. 2002;99(18):11896-11901.

56. Barter PJ, Brewer HB Jr, Chapman MJ, Hennekens 
CH, Rader DJ, Tall AR. Cholesteryl ester transfer protein: a novel target for raising HDL and inhibiting atherosclerosis. Arterioscler Thromb Vasc Biol. 2003;23(2):160-167.

57. Erbay E, et al. Reducing endoplasmic reticulum stress through a macrophage lipid chaperone alleviates atherosclerosis. Nat Med. 2009;15(12):1383-1391.

58. Feig JE, et al. LXR promotes the maximal egress of monocyte-derived cells from mouse aortic plaques during atherosclerosis regression. J Clin Invest. 2010;120(12):4415-4424.

59. Murphy AJ, et al. ApoE regulates hematopoietic stem cell proliferation, monocytosis, and monocyte accumulation in atherosclerotic lesions in mice. J Clin Invest. 2011;121(10):4138-4149.

60. Yvan-Charvet L, et al. ATP-binding cassette transporters and HDL suppress hematopoietic stem cell proliferation. Science. 2010;328(5986):1689-1693.
61. Yasuda T, Grillot D, Billheimer JT, Briand F, Delerive P, Huet S, Rader DJ. Tissue-specific liver X receptor activation promotes macrophage reverse cholesterol transport in vivo. Arterioscler Thromb Vasc Biol. 2010;30(4):781-786.

62. Kalaany NY, et al. LXRs regulate the balance between fat storage and oxidation. Cell Metab. 2005; 1(4):231-244.

63. Turley SD, Daggy BP, Dietschy JM. Psyllium augments the cholesterol-lowering action of cholestyramine in hamsters by enhancing sterol loss from the liver. Gastroenterology. 1994;107(2):444-452.

64. Turley SD, Daggy BP, Dietschy JM. Effect of feeding psyllium and cholestyramine in combination on low density lipoprotein metabolism and fecal bile acid excretion in hamsters with dietary-induced hypercholesterolemia. J Cardiovasc Pharmacol. 1996;27(1):71-79.
65. Yu L, et al. Overexpression of ABCG5 and ABCG8 promotes biliary cholesterol secretion and reduces fractional absorption of dietary cholesterol. J Clin Invest. 2002;110(5):671-680.

66. Lee YK, et al. Liver receptor homolog-1 regulates bile acid homeostasis but is not essential for feedback regulation of bile acid synthesis. Mol Endocrinol. 2008;22(6):1345-1356.

67. Bookout AL, Mangelsdorf DJ. Quantitative realtime PCR protocol for analysis of nuclear receptor signaling pathways. Nucl Recept Signal. 2003; 1:e012.

68. Tangirala RK, Rubin EM, Palinski W. Quantitation of atherosclerosis in murine models: correlation between lesions in the aortic origin and in the entire aorta, and differences in the extent of lesions between sexes in LDL receptor-deficient and apolipoprotein E-deficient mice. J Lipid Res. 1995;36(11):2320-2328. 